\title{
Renal Vein Thrombosis secondary to pyelonephritis: Case presentation
}

\author{
Anusha Skandini Ganeshan*, Angel Alberto Calderon Chongo \\ Internal Medicine Department, Seychelles Hospital, Seychelles \\ *Corresponding Author: Anusha Skandini Ganeshan: a_skandini2000@yahoo.com
}

Citation: Ganeshan A. S., Chongo A. A. C. (2017) Renal Vein Thrombosis secondary to pyelonephritis: Case presentation. Open Science Journal 2(2).

Received: $17^{\text {th }}$ January 2017

Accepted: $7^{\text {th }}$ March 2017

Published: $10^{\text {th }}$ April 2017

Copyright:@ 2016 This is an open access article under the terms of the Creative Commons Attribution License, which permits unrestricted use, distribution, and reproduction in any medium, provided the original author and source are credited.

Funding: The author(s) received no specific funding for this work

Competing Interests: The authors have declared that no competing interests exist.

\begin{abstract}
The Renal Vein Thrombosis (RVT) is a rare pathology in adults as compared to children. It is usually present as a complication of other conditions as sepsis, hypercoagulable state, and malignancy. We present a male of age of 36 years, who suffered from RVT following pyelonephritis. Following all investigations, including blood tests, Computed Tomography (CT) of abdomen and CT angiogram, the concluding diagnosis of left RVT was made. Subsequent to confirmation of diagnosis, he was commenced an anticoagulation therapy with the low-molecularweight heparin and warfarin. Consequently, the patient was discharged home with regular follow-ups on an outpatient basis with PT/INR checks. After 3 months, a control CT renal angiogram was performed, which revealed complete resolution of the Left RVT. In conclusion of this patient case, it can be stated that RVT is a rare condition which can have a good outcome with early diagnosis and subsequent appropriate anticoagulant therapy. In a view of this case, the review of the topic was made.
\end{abstract}

Keywords: Renal Vein Thrombosis (RVT), Anti-Coagulant therapy, Warfarin, Pyelonephritis, CT Angiogram. 


\section{Introduction}

Renal Vein Thrombosis (RVT) is the formation of a clot in the vein that drains blood from the kidneys, ultimately leading to a reduction in the drainage of one or both kidneys and the possible migration of the clot to other parts of the body, leading to complications as Pulmonary embolism for example [1]. RVT may appear as an acute condition or a chronic condition or may be undiagnosed due to the lack of symptoms [2].

This is a rare condition in adults, however, it is more frequent in neonates and children, appearing in 5 of 100000 births; more than $40 \%$ of all thrombotic manifestations in this age group are symptomatic RVTs [3].

Studies in the United States have shown the prevalence RVT in adults is highly variable ranging from $5 \%$ to $62 \%[4,5]$. In patients who have undergone a renal transplant, the risk of developing RVT is $0.6 \%$ to $6 \%$ [1]. Among adults, males are reported to be more affected than females according to some studies. In these cases, bilateral renal veins are frequently involved. However, in cases of unilateral RVT, the left renal vein is reported to be more commonly affected than the right one [2].

The possible complications of RVT include recurrent thromboembolic phenomena [1], hypertension [2], renal failure, problems specific to a particular cause (such as graft failure after renal transplantation) [1].

\section{Case report}

A 36-year-old man who is a known Epileptic since childhood, on Carbamazepine and Phenobarbitone; and recently diagnosed with the iron deficiency anaemia on treatment, presented with complaints about acute onset of left sided abdominal and loin pain early that morning; he reported to have fever for three days, vomited seven times since early morning, and experienced slight burning micturition. He denied any recent trauma or injury. His bowel habit was normal.

General examination revealed the fever of $38.6^{\circ} \mathrm{C}$; blood pressure of $133 / 65 \mathrm{mmHg}$; other vital signs were within normal range. The clinical examination that was significant to note was mild pallor, mucosa mild dry, and abdominal tenderness in the left-side abdomen (flank) region, with positive Pasternacki's sign on the left; the rest of the abdomen was soft.

\section{Management}

He was initially diagnosed with left pyelonephritis and started treatment with IV fluids, analgesics, and intravenous antibiotics (ceftriaxone).

\section{Investigations}

$>$ Ultrasound of Abdomen: free intraperitoneal fluid noted in Morison's pouch \& pelvis. 
$>$ Abdominal X-ray and chest X-ray - normal, no air under the diaphragm found, no air-fluid levels to note.

$>$ WBC $15.0 \times 10^{3} \mathrm{uL}$; Hgb $9.7 \mathrm{~g} / \mathrm{dl}$; Platelet $298 \times 10^{3} \mathrm{uL}$; MCV $52.8 \mathrm{fL}$

$>$ Liver and renal parameters: within normal limits (Cr 90);

$>$ Amylase 40;

$>$ C-Reactive Protein (CRP): $179.6 \mathrm{mg} / \mathrm{l}$

$>$ Urine micro: white blood cell 10/HPF; red blood cell $5 / \mathrm{HPF}$; + few bacterial organisms seen

$>$ CT ABDOMEN (1st): free fluid noted in perihepatic area, both anterior perirenal space and pelvis with left renal hydronephrosis.

On succeeding days, patient's fever subsided, however, he had the persistent left loin pain. Thus, a repeat of abdominal CT with renal angiogram was requested, which showed Left Renal Vein Thrombosis as a cause of the left renal parenchymal oedema; left kidney upper lobe hypoperfusion, suggestive of pyelonephritis; Interval reduction on perirenal and intraperitoneal fluid compared to previous CT scan. Delayed contrast secretion and excretion noted.

\section{Diagnosis}

Left Renal Vein Thrombosis with left Pyelonephritis.

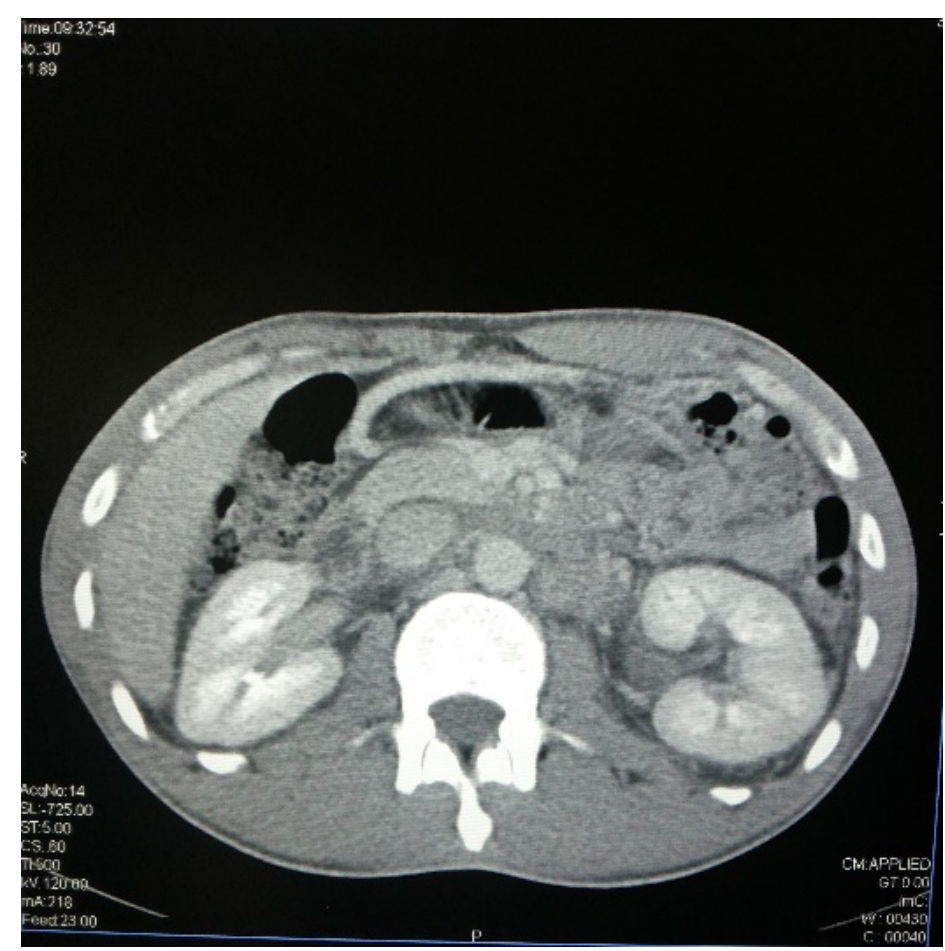

Figure 1

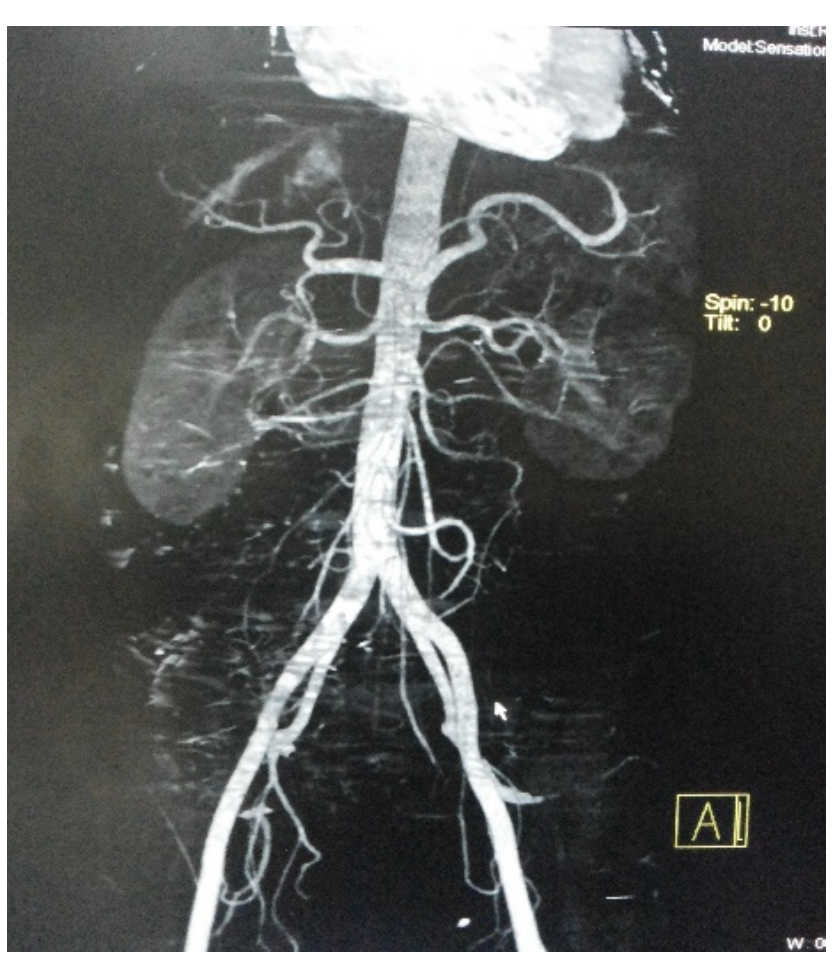

Figure 2 


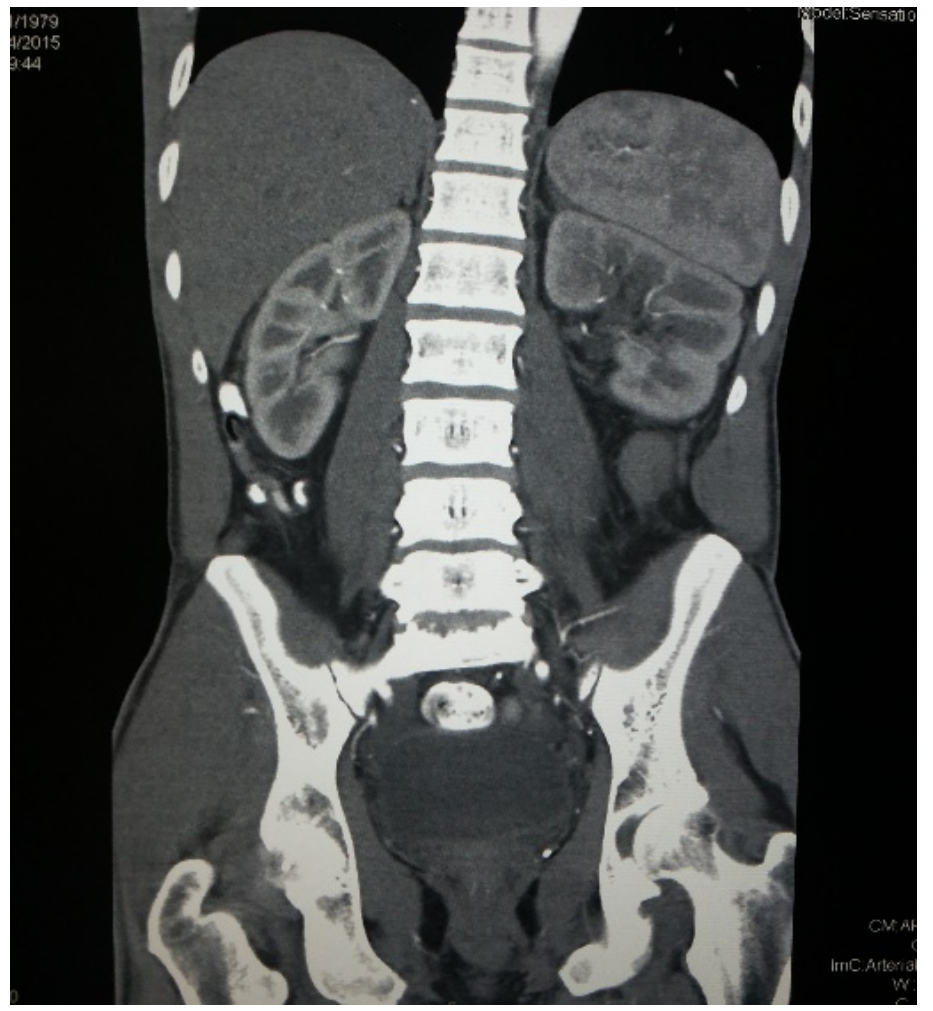

Figure 3

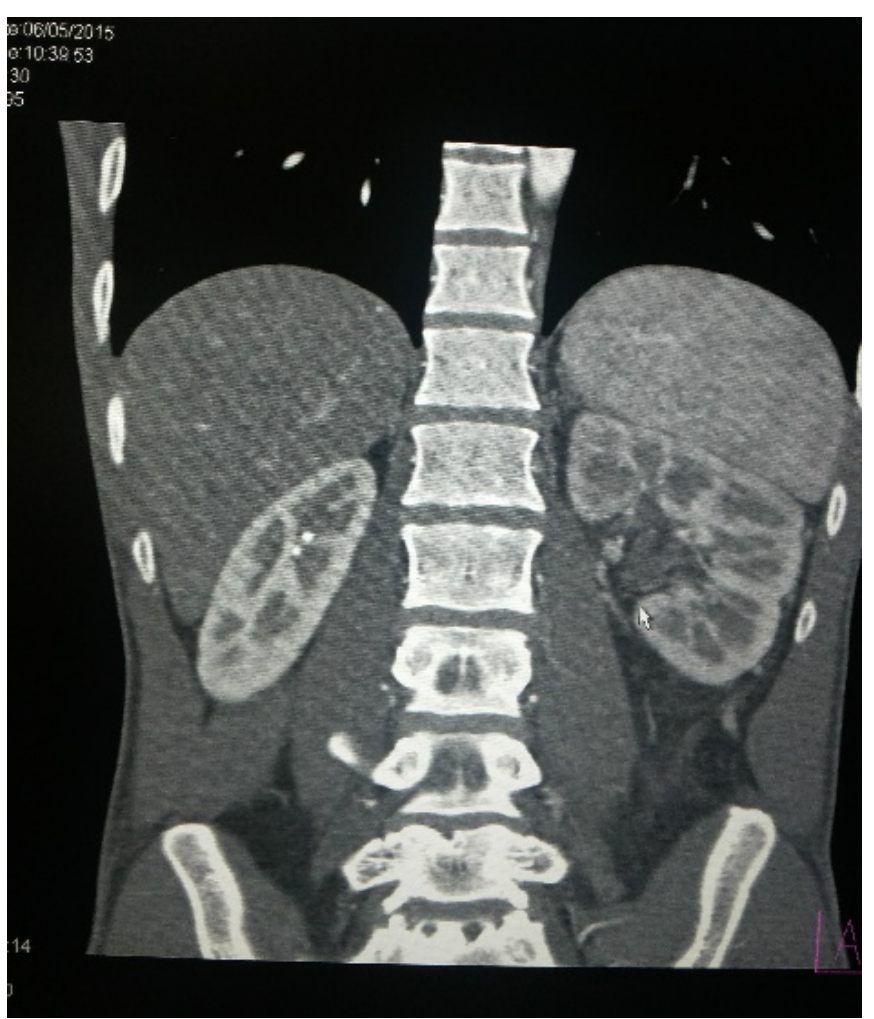

Figure 4

The above images of this patient's CT Angiogram in axial view (figures 1 \& 2) and coronal view (figures 3 \& 4 ) respectively illustrates: Left Renal Vein Thrombosis causing the left renal parenchymal oedema; note that on comparison the contrast flows freely through the right renal vein.

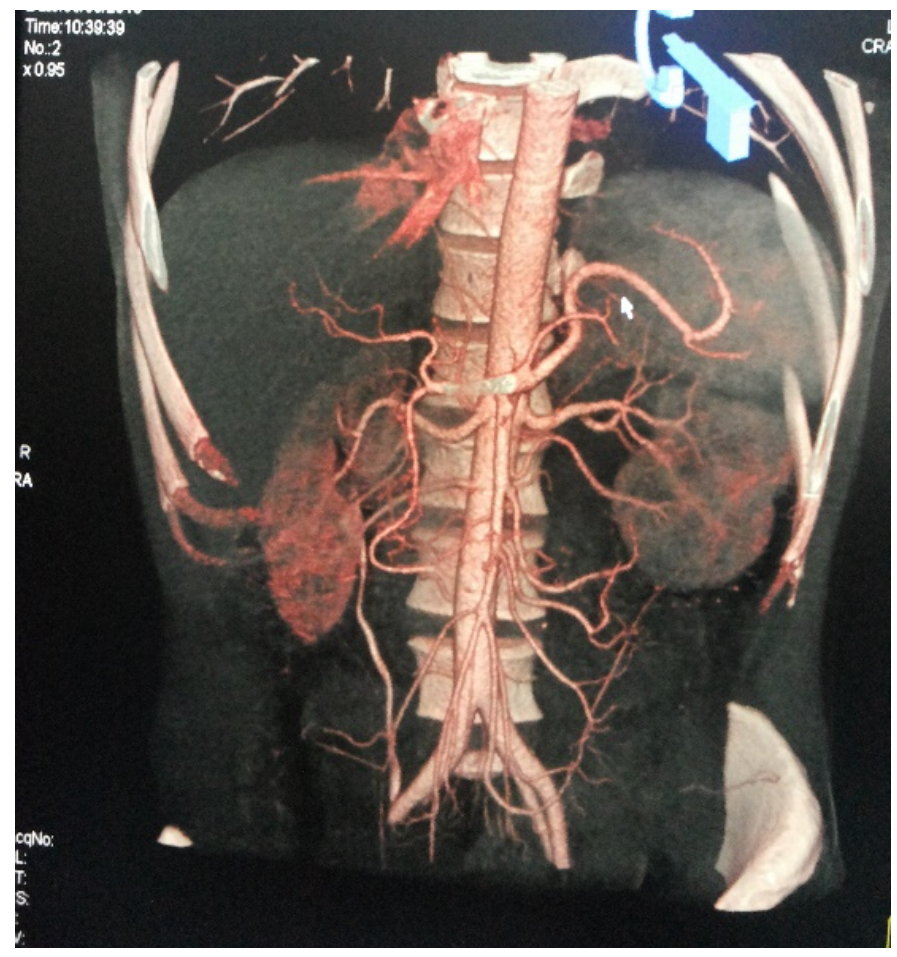

Figure 5

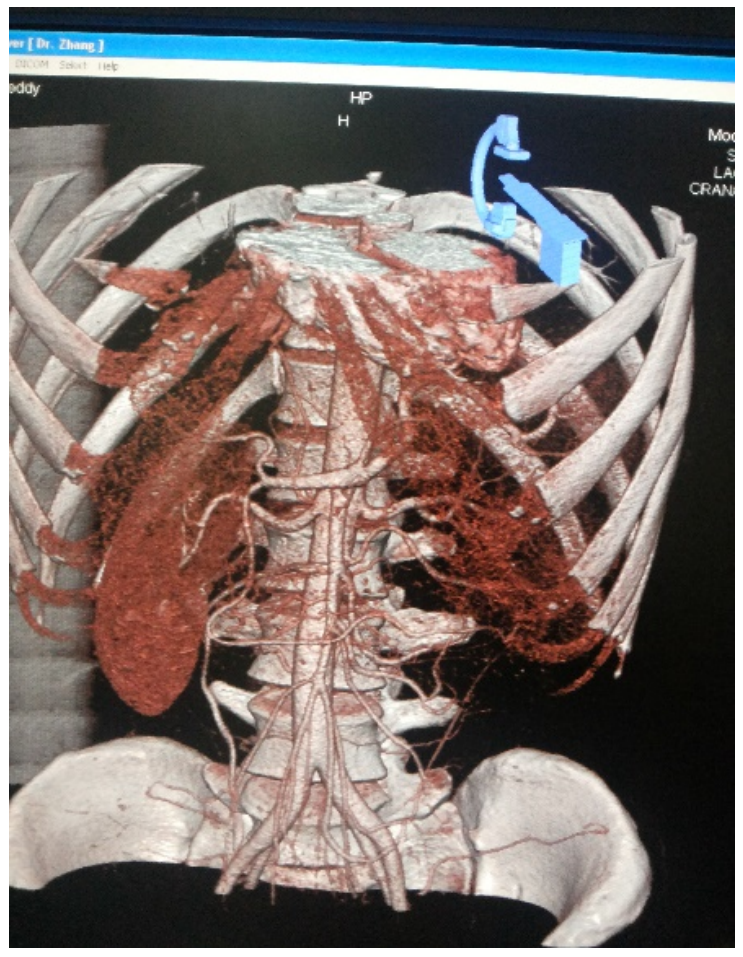

Figure 6 
The above figures $5 \& 6$ represent CT Angiogram 3D reconstructed images that clearly illustrate the presence of Left RVT in 1st image and subsequent left kidney upper lobe hypoperfusion illustrated in both images with delayed contrast secretion and excretion.

\section{Further Management}

After discovering the Left RVT, the patient was commenced on the lowmolecular-weight heparin (LMWH) and warfarin. In the successive days in the hospital, it was noted that patient's INR remained below the therapeutic range, due to the interference of both of patient's antiepileptic medications (phenobarbitone and carbamazepine) with warfarin. Therefore, both his antiepileptics were gradually weaned off and he started with sodium valproate with dose adjustment.

Subsequently, the therapeutic INR was achieved and the patient was discharged with follow-up of PT/INR. On serial checks of his renal parameters, it remained normal at all times.

\section{Follow-up}

After a period of three months on warfarin, a repeat of abdominal CT with renal angiogram was done, which revealed complete resolution of left RVT. Thus, the warfarin therapy was discontinued and he was commenced on aspirin. He is still on regular follow-ups to date.

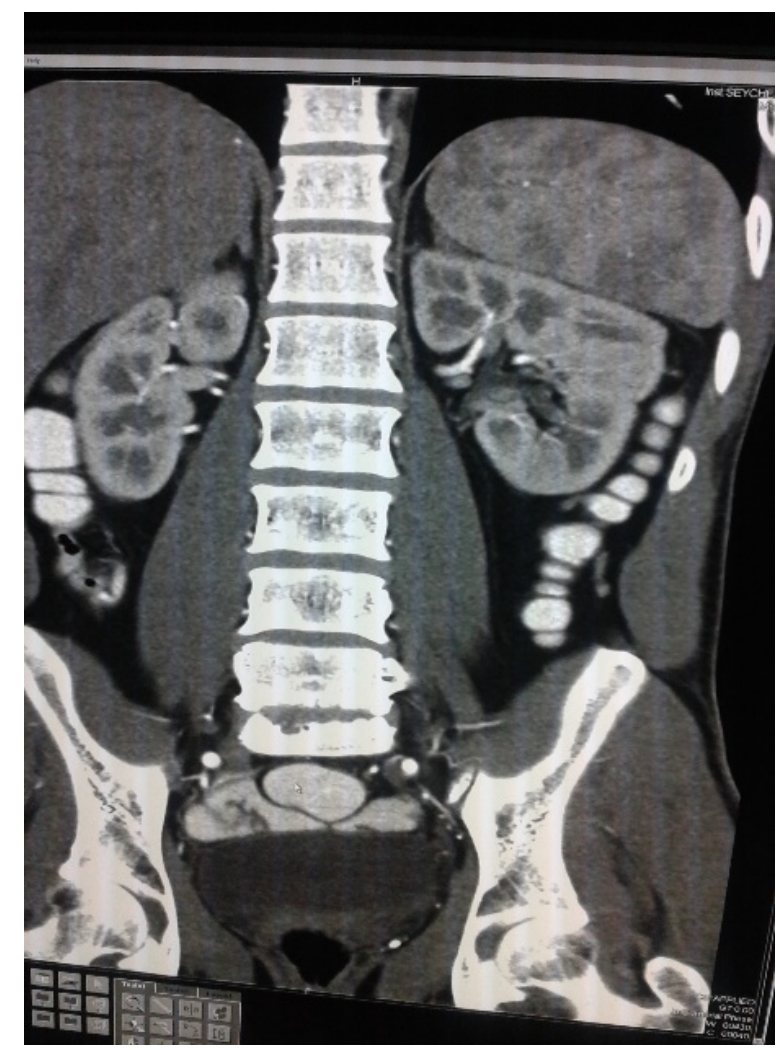

Figure 7 
On the figure 7 we can see the image of patient's CT angiogram after the anticoagulation therapy, which indicates the clear flow of contrast through the left renal vein, indicating the resolution of RVT.

\section{Discussion}

Renal Vein Thrombosis (RVT) is a rare condition [3,6]. In Seychelles, in clinical practice, there is a high rate of hypercoaguable states with numerous cases of Deep Vein Thrombosis (DVT) and Pulmonary Embolism (PE) reported yearly, in most cases suspected to be a secondary to Protein $\mathrm{C}$ and $\mathrm{S}$ deficiencies. Although to date there is no publication about this, there is, however, an ongoing study in Seychelles. In a study on causes of a sudden death in Seychelles, it has been found that the second leading cause of a sudden death in Seychelles is PE with DVT [7]. Nonetheless, thus far over the past 10 years, there have not been any reported cases of RVT except for the above one.

\section{Etiology}

RVT can be caused by numerous etiologies, including hypercoaguable state such as in urinary sepsis, nephrotic syndrome (which may result from membranoproliferative glomerulonephritis, minimal change disease, rapidly progressive glomerulonephritis, amyloid, focal sclerosis, or lupus nephritis) [4]; Protein C, protein S or antithrombin deficiency, antiphospholipid syndrome $[2,4]$, factor V Leiden mutation, prothrombin G20210A mutation [8]; stasis such as dehydration, haemorrhage, post-renal transplant or retroperitoneal malignancy with compression of renal vein; other less common causes include damage to endothelial cells secondary to conditions as trauma or infiltrative tumour, or vasculitis; SLE $[2,4,8]$.

Some studies have shown that RVT is more common in males than in females, and in cases of unilateral RVT, the left renal vein is more affected than the right $[2,9]$, thus the same has been proven with the above male patient in this study.

\section{Clinical manifestation}

The clinical manifestations of RVT are variable depending on etiology, but common manifestations include acute flank pain, haematuria (frank/ microscopic), nausea, vomiting, and fever [3,9,10]. These clinical manifestations were present in the patient case discussed above.

When the etiology is a hypercoagulability disorder, signs of venous thromboembolic disorders as DVT (present as acute unilateral lower limb/calf tenderness, oedema [11]), or PE may occur concurrently, (with variable symptoms such as tachycardia, shortness of breath, hypoxia, shock, hypotension, chest pain, and haemoptysis [12]), which is a life-threatening condition if untreated [1,11]. In cases of nephrotic syndrome, increased proteinuria, pedal oedema, and renal enlargement are present. In cases of bilateral RVT clinical presentation may be with acute renal failure, with decreased urine output $[8,9,10]$. 
In renal carcinoma presentation, symptoms of hematuria and weight loss may predominate [8].

In infants and neonates, common causes of RVT primarily include dehydration and sepsis [13], other causes include polycythaemia, sickle cell disease, maternal diabetes, and indwelling umbilical venous catheters [14]. Male children with left-sided RVT may lead to gonadal vein thrombosis with painful swelling of the left testis and varicocele formation [9].

\section{Investigations}

There are no specific laboratory tests that can confirm an RVT. The workup in done primarily based on the assessment of patient's clinical presentation, history, and physical examination. RVT is only diagnosed by imaging studies.

Upon presentation, basic blood workup is carried out, which is vital to identify the cause of illness and rule out any concurrent conditions, which will contribute to the respective supportive management of the patient.

\section{Blood tests/ urine tests:}

$>$ Complete blood count (to rule out sepsis, anaemia, polycythemia, or thrombocytopenia $[3,15,16])$.

$>$ Serum creatinine and blood urea nitrogen (BUN) indicate acute renal failure; Albumin levels for hypoalbuminemia [3,4,15].

$>$ Erythrocyte sedimentation rate (ESR) and C-reactive protein (CRP) levels (elevated in acute systemic inflammatory/infectious states) [6].

> Urinalysis for microscopic hematuria, which is common finding $[3,8,15]$; proteinuria ( $>3 \mathrm{~g} /$ day indicates Nephrotic syndrome).

$>$ Hypercoagulability disorders should be initiated if all above normal, which includes: $[8,15]$ which includes: prothrombotic analysis for genetic defects such as prothrombin 20210 gene mutation, concentrations (deficiency) of protein $\mathrm{C} / \mathrm{S}$ and antithrombin; Factor V Leiden mutation, which causes resistance to activated protein $\mathrm{C}$, antithrombin deficiency [17]; anticardiolipin antibodies [18].

$>$ Antiphospholipid antibodies; Cholesterol levels for hypercholesterolemia $[4,16]$.

Based on this context, in the above patient case, who was diagnosed with pyelonephritis as the cause of left RVT, the significant findings in blood tests include the following: the elevated serum WBC count of $15 \times 10^{3}$, and CRP significantly elevated 179.6 (normal range $<5$ ). Urine tests also exhibit some significant findings:the presence of WBC, with microscopic hematuria and a few bacterial organisms, though a specific bacterial organism was not isolated.

\section{Imaging studies}

Imaging studies finding will vary according to the time of onset of renal vein occlusion and the degree of occlusion [1,2].

$>$ Abdominal ultrasound (US): can reveal renal enlargement with hypoechoic cortex from oedema (early phase). Subsequently, however, there is a gradual reduction in the renal size over the next few weeks and later a renal atrophy and increased echogenicity (late sign) [2,14]. 
> Color Doppler Ultrasound: Doppler findings include reversal of arterial diastolic flow, absent venous flow, visualization of thrombus within the lumen, and high resistance in the renal artery with elevated resistive index [14].

However, ultrasonography is usually not sensitive enough to assist in making the diagnosis [4] as it carries high false-negative and false-positive rates.

\section{Computed Tomography (CT) Angiography}

It is currently the investigation of choice in diagnosing RVT. It is noninvasive, fast, [1], and available in most hospitals. Angiography is carried out by administration of an intravenous radiocontrast agent, which may be nephrotoxic. Thus, serum electrolytes and urinalysis are done and confirm deterioration of renal function [8] prior and post-procedure.

\section{Radiological finding}

The thrombosis is observed as a filling defect during venous phase imaging following intravenous contrast. Changes in the attenuation, either focal or diffuse, may be present in the end organ kidney due to perfusion abnormalities [14]. CT scan also provide information on renal enlargement and other possible causes of RVT as renal tumours and other renal/ pararenal pathologies $[1,2]$.

\section{Magnetic Resonance Angiography (MRA)}

Is a non-invasive, fast, though more costly imaging study [1] and has limited availability. Its major benefit is the avoidance of radiation and IV contrast material [4, 14]. MRI also may help to detect RVT and the presence of a tumour; [4] similarly the anatomic variants [2].

\section{Inferior venocavography (IVC)}

Selective venography can be used to rule out the diagnoses of RVT [4]. Finding that is indicative of RVT includes a lack of washout of contrast or obvious filling defects due to the presence of thrombus. However, venography is invasive, involves higher radiation exposure and injection of iodinated contrast, and carries a risk of de novo RVT due to venous injury [2].

\section{Renal biopsy}

Renal biopsy is not routinely performed, nonetheless is essential in evaluating patients with nephrotic syndrome with an underlying primary renal disease. Membranous nephropathy is the most common finding [4].

For the case discussed above, the ultrasound was not conclusive. Thus, as discussed, further investigation was done, and confirmatory diagnosis of left RVT was made by CT Angiogram, images of which are illustrated above.

\section{Management}

The management of patients with RVT should reflect the underlying causative factors [9]. Treatment includes intravenous (IV) fluid rehydration in 
cases of dehydration, treating a co-existing focal/ systemic infection with IV antibiotics, prevention or treatment of a urinary infection [3,15]. Measures employed to treat nephrotic syndrome may include steroids and immunosuppressive therapy, along with symptomatic treatment as diuretics and angiotensin-converting enzyme inhibitors (ACEIs) or angiotensin II receptor blockers (ARBs) to decrease proteinuria in nephrotic syndrome [4].

\section{Anticoagulation}

In the past, the treatment of RVT was primarily surgical involving thrombectomy or nephrectomy [2]. But it is very invasive and many complications can occur. Over the past decades, treatment has shifted its focus from surgical intervention to medical treatments that include intravenous and oral anticoagulants [1]. Anticoagulation treatment options include heparin or lowmolecular-weight heparin injections (LMWH) and long-term anticoagulation oral warfarin [8,15]. Anticoagulation treatment should be started immediately if no invasive intervention is planned, as this treatment minimizes the risk of new thrombi, promotes recanalization of vessels with existing clots, and improves renal function [8].

There is little evidence to guide the duration of anticoagulation treatment [9]. Some studies say, depending on severity and cause of RVT, anticoagulation therapy should be continued for at least 6 to 12 months, and if a hypercoagulability disorder (example: persistent nephrotic syndrome) is present, then indefinite[1,8].

\section{Thrombolysis or percutaneous catheter for thrombectomy [8]}

The indication for thrombolysis in the setting of RVT is unclear. In the case of PE from other causes, thrombolytics are indicated in the setting of pulmonary hypertension (as found during examination or discovered by echocardiography). Catheter-based techniques for rapid delivery of thrombolytics in the setting of acute or refractory RVT have been described [4].

\section{Surgical treatment}

Although it is rarely indicated today [4], but should be considered in patients with acute bilateral renal vein thrombosis and acute kidney injury who cannot be treated with percutaneous catheter thrombectomy and/or thrombolysis [8] or if anticoagulation is contraindicated.

$>$ Inferior vena caval filters: may be used in this instance and also may be necessary in the event of failure of medical therapy for recurrent thromboembolism[4].

$>$ Surgery with Nephrectomy: is done only if infarction is total (in certain cases) or if the underlying disorder warrants it [8] such as RVT of Renal cell carcinoma in early-stage disease [14].

In the patient case discussed above, a timely management of anticoagulation with LMWH and warfarin, followed by maintenance of the therapeutic range of INR with adjustment of warfarin on follow-up, ensured that patient did not suffer from any complications and made a complete recovery with resolution of RVT in the progressive months. 


\section{Complications}

$>$ Pulmonary embolism $(\mathrm{PE})$

$>$ Acute kidney injuries, such as renal papillary necrosis and acute renal failure

$>$ Hypertension [15]

$>$ Renal atrophy with end-stage renal disease [14]

$>$ Recurrent thromboembolism [4]

$>$ Complications related to renal transplant patients as graft failure [1]

\section{Mortality/Morbidity}

Mortality due to RVT is rare and usually related to complications such as pulmonary embolism, especially in the presence of chronic RVT [11]; Other complications include those due to nephrotic syndrome or a malignant tumour $[8,9]$, or in the setting of transplantation, RVT may lead to failure of the graft [1].

Prognosis of the condition, however, has improved significantly over the last few decades due to the availability of better diagnostic tests, the vast possibility of timely dialysis, and ensuing use and availability of anticoagulation therapy [2].

\section{Conclusion}

RVT is thus far an uncommon condition, especially in adults. It may be stated that RVT may go undiagnosed due to the lack of symptoms in patients or an overlooked diagnosis in some clinical settings. Nonetheless, as many studies have proven, it is vital to diagnose RVT, especially in a setting of bilateral RVT, as complications of the disease, if undiagnosed, may be fatal, especially in a view of acute renal failure and $\mathrm{PE}$.

The best diagnostic method used to diagnose RVT is by far CT Angiography with high accuracy as compared to Doppler ultrasound. Patients can be effectively treated with anticoagulants, provided no contraindications. But one must bear in mind the possible complications of anticoagulants (or anticoagulation therapy), and hence patients are followed-up on regular basis to ensure the therapeutic INR range is always maintained.

Furthermore, on timely diagnosis of RVT, when it is effectively treated the prognosis and outcome is very good, as in the case discussed in this article. Prognosis for patients with renal cell carcinoma or nephrotic syndrome may vary depending on the course and complications of the primary illness as opposed to RVT alone. Therefore, to have a good outcome for patients with RVT, early diagnosis and appropriate timely management is imperative. 


\section{References}

1. Renal vein Thrombosis; https://medlineplus.gov/ency/article/000513.htm, 27 May 2016

2. Renal vein Thrombosis, M. Asghar, K Ahmed et Al; Eur J Endovasc Surg 34, 217-223 (2007).

3. Renal venous thrombosis in neonates: prothrombotic risk factors and long-term follow-up; Andrea Kosch, Eberhard Kuwertz-Bro king et Al; From www.bloodjournal.org by guest on September 24, 2016..

4. Renal Vein Thrombosis; Author: Igor A Laskowski, MD; Chief Editor: Vincent Lopez Rowe, MD; http://emedicine.medscape.com/article/460752-overview?src=refgatesrc1 \#a6; September 01,2016

5. Renal Vein Thrombosis; Nephrology Grand Rounds 10/18/11; Nicola Sumorok.

6. Acute renal vein thrombosis and nephrotic syndrome in the setting of retroperitoneal fibrosis; M.W. Wilson and M.W. Milks; Oxford Medical Case Reports, 2015 , 309-310, May 28, 2015

7. $\quad$ P. Zhao 34 et al; Journal of Forensic and Legal Medicine 37 (2016) 33e38; www.elsevier.com/locate/jflm

8. Renal Vein Thrombosis Zhiwei Zhang, MD; July 2016; http://www.merckmanuals.com/professional/genitourinary-disorders/renovasculardisorders/renal-vein-thrombosis

9. Guidelines on the investigation and management of venous thrombosis at unusual sites; Campbell Tait, Trevor Baglin et al; British Journal of Haematology, 2012, 159,28-38; http://www.bcshguidelines.com/documents/VTE_Unusual_Sites_BJH_Oct12.pdf

10. Acute Bilateral Renal Vein Thrombosis; Qi Qian, MD, Nathan A. Saucier, MD, and Bernard F. King, MD; American Journal of Kidney Diseases, Vol 54, No 5 (November), 2009: pp 975-978

11. Venous Thromboembolism (Blood Clots); February 24, 2016; http://www.cdc.gov/ncbddd/dvt/facts.html

12. A Venous \& Arterial Thrombosis Resource For Physicians; 20th September 2016; 2016 Bayer Pharma AG; https://www.thrombosisadviser.com/en/vte-treatment/pulmonary-embolism/

13. Renal venous thrombosis in Newborns; Margaret L. Fallon, M.B; http://adc.bmj.com/ on September 24, 2016

14. Renal vein thrombosis; Dr Henry Knipe and Radswiki et al; 2016 Radiopaedia.org; https://radiopaedia.org/articles/renal-vein-thrombosis

15. Renal venous thrombosis in neonates; Moudgil A; Curr Pediatr Rev. 2014;10(2):101-6; https://www.ncbi.nlm.nih.gov/pubmed/25088263

16. Nephrotic Syndrome (NS); What Is Renal Vein Thrombosis?; Simeon Margolis, M.D., Ph.D; 02 Feb 2015; http://www.healthcommunities.com/nephrotic-syndrome-ns/what-is-renal-veinthrombosis.shtml

17. Perinatal renal venous thrombosis: presenting renal length predicts outcome; P J D Winyard, T Bharucha et al; Arch Dis Child Fetal Neonatal Ed. 2006 Jul; https://www.ncbi.nlm.nih.gov/pmc/articles/PMC2672730/

18. Overview of Thrombotic Disorders By Joel L. Moake, MD; 2016 Merck Sharp \& Dohme Corp; http://www.merckmanuals.com/professional/hematology-and-oncology/thromboticdisorders/overview-of-thrombotic-disorders. 